\title{
Onset of Bénard-Marangoni ferroconvection with a convective surface boundary condition: The effects of cubic temperature profile and MFD viscosity
}

\author{
C.E. Nanjundappa ${ }^{\text {a, } *}$, I.S. Shivakumara ${ }^{\text {b }}$, B. Savitha ${ }^{c}$ \\ a Department of Mathematics, Dr. Ambedkar Institute of Technology, Bangalore 560 056, India \\ ${ }^{\mathrm{b}}$ UGC-Centre for Advanced Studies in Fluid Mechanics, Department of Mathematics, Bangalore University, Bangalore 560001 , India \\ c Department of Mathematics, Rajarajeswari College of Engineering, Bangalore 560 074, India
}

\section{A R T I C L E I N F O}

Available online 2 December 2013

\section{Keywords:}

Bénard-Marangoni ferroconvection

Cubic temperature profile

MFD viscosity

Galerkin technique

Convective surface boundary condition

\begin{abstract}
A B S T R A C T
The combined effects of basic cubic temperature profiles and magnetic field dependent (MFD) viscosity on the onset of Bénard-Marangoni convection in a ferrofluid layer are studied. The lower boundary is rigid-isothermal, while the upper free boundary open to the atmosphere is flat and subject to a general thermal boundary condition. The Galerkin technique is employed to extract the critical stability parameters numerically. The results indicate that the basic cubic temperature profiles have a profound influence on the stability characteristics of the system and can be effectively used to either suppress or augment the onset of Bénard-Marangoni ferroconvection. Besides, increasing the magnetic Rayleigh number and the nonlinearity of magnetization hastens, while an increase in the Biot number and MFD viscosity parameter delays the onset of Bénard-Marangoni ferroconvection. The existing results in the literature are obtained as particular cases from the present study.
\end{abstract}

() 2013 Elsevier Ltd. All rights reserved.

\section{Introduction}

Ferrofluids are stable colloidal suspensions of magnetic nano-particles dispersed in a carrier liquid. In the absence of an external magnetic field the magnetic moments of the particles are randomly orientated and there is no net macroscopic magnetization. In an external magnetic field, however, the magnetic moments of particles easily orient and a large (induced) magnetization prevails. There are two additional features in ferrofluids not found in ordinary fluids, the Kelvin force and the body couple [1]. In addition, in an external magnetic field, a ferrofluid exhibits additional rheological properties such as a field-dependent viscosity, special adhesion properties, and a non-Newtonian behavior, among others [2]. The theory of thermal convective instability in a ferrofluid layer began with Finlayson [3] and extensively continued over the years ([4-6]). Recently, Shivakumara et al. [7] have investigated the onset of thermogravitational convection in a horizontal ferrofluid layer with viscosity depending exponentially on temperature.

On the other hand, if the surface of a ferrofluid layer is free and open to the atmosphere then convection can also be induced by temperature dependent surface tension forces at the free surface known as Marangoni ferroconvection. In view of the fact that heat transfer is greatly enhanced due to convection, Marangoni ferroconvection offers new possibilities for application in cooling of motors in space, loudspeakers, transmission

\footnotetext{
Communicated by A.R. Balakrishnan and T. Basak.

* Corresponding author.

E-mail address: cenanju@hotmail.com (C.E. Nanjundappa).
}

lines and other equipments in micro-gravity environment where a magnetic field is already present. In most of the cases, the combined effect of buoyancy and surface tension forces on convective instability in a ferrofluid layer becomes important. Realizing these aspects, a limited number of studies have addressed the effect of surface tension forces on ferroconvection in a horizontal ferrofluid layer. Linear and nonlinear stability of combined buoyancy-surface tension effects in a ferrofluid layer heated from below has been analyzed by Qin and Kaloni [8]. The linear stability analysis of a layer of a magnetic fluid with a deformable free surface which is heated uniformly from below and subject to a vertical magnetic field has been analyzed considering the temperature dependence of the surface tension and buoyancy by Weilepp and Brand [9]. Odenbach [10] has investigated experimentally the stability of a free surface of a magnetic fluid subjected to a magnetic field parallel to the fluid surface under strongly reduced gravity. The Bénard-Marangoni problem of ferrofluids has been studied for different situations in Refs. [11-13]. Idris and Hashim [14] have investigated the instability of Bénard-Marangoni convection in a horizontal layer of ferrofluid under the influence of a linear feedback control and cubic temperature profile. Nanjundappa et al. [15] have studied the effect of internal heat generation on the onset of Bénard-Marangoni convection in a horizontal ferrofluid layer heated from below in the presence of a uniform vertical magnetic field. Recently, the effect of temperature dependent viscosity on Marangoni ferroconvection is considered by Nanjundappa et al. [16]. In the contemporary heat transfer research involving ferrofluids it is imperative to understand the mechanisms for control (suppression or augmentation) of Bénard-Marangoni ferroconvection which is 
useful in materials science processing under microgravity conditions. One of the effective mechanisms is to maintain a non-uniform basic temperature gradient across the ferrofluid layer. The main objective of the present study is to consider this aspect and analyze different forms of basic cubic temperature profiles on the onset of BénardMarangoni convection in a horizontal ferrofluid layer in the presence of a uniform vertical magnetic field. In investigating the problem, the effect of magnetic field dependent viscosity is also considered as the viscosity of the ferrofluid varies significantly with magnetic field. The lower boundary of the ferrofluid layer is rigid-isothermal, while the upper boundary is free, non-deformable and subject to a general thermal boundary condition. Moreover, at the upper free boundary the surface tension effects due to temperature are considered. The resulting eigenvalue problem is solved numerically by employing the Galerkin technique with Chebyshev polynomials as trial functions.

\section{Mathematical formulation}

We consider a Boussinesq ferrofluid layer of thickness $d$ in the presence of a uniform vertical magnetic field $H_{0}$. The lower and upper boundaries are maintained at constant but different temperatures $T_{0}$ and $T_{1}\left(<T_{0}\right)$ respectively. A Cartesian co-ordinate system $(\mathrm{x}, \mathrm{y}, \mathrm{z})$ is used with the origin at the bottom of the porous layer and the $z$-axis is directed vertically upward. The gravitational force $(0,0,-\mathrm{g})$ acts in the negative z-direction. Experimentally, it has been demonstrated that the viscosity $\eta$ of ferrofluids varies significantly with respect to the magnetic field (Rosensweig et al. [17]). As a first approximation, for small field variation, the linear variation of viscosity with magnetic field has been considered in the form $\eta=\eta_{0}(1+\vec{\delta} \cdot \vec{B})$, where $\vec{\delta}$ is the variation coefficient of magnetic field dependent viscosity and is considered to be isotropic (Vaidyanathan et al. [18]), and $\eta_{0}$ is the viscosity of the fluid in the absence of magnetic field. Several investigators in the past have followed this assumption in the study of Marangoni convection and the free surface is assumed to be non-deformable (zero capillary number). At the upper free surface, the surface tension $\sigma$ is assumed to vary linearly with temperature in the form

$\sigma=\sigma_{0}-\sigma_{T}\left(T-T_{0}\right)$,

where, $\sigma_{0}$ is the unperturbed value and $-\sigma_{T}$ is the rate of change of surface tension with temperature $T$.

The governing equations under the Oberbeck-Boussinesq approximation are given by the following:

$\nabla \cdot \vec{q}=0$

$\rho_{0}\left[\frac{\partial \vec{q}}{\partial t}+(\vec{q} \cdot \nabla) \vec{q}\right]=-\nabla \mathrm{p}+\rho \vec{g}+\nabla \cdot\left[\eta\left(\nabla \vec{q}+\nabla \vec{q}^{T}\right)\right]$

$\left[\rho_{0} C_{V, H}-\mu_{0} \vec{H} \cdot\left(\frac{\partial \vec{M}}{\partial T}\right)_{V, H}\right] \frac{D T}{D t}+\mu_{0} T\left(\frac{\partial \vec{M}}{\partial T}\right)_{V, H} \cdot \frac{D \vec{H}}{D t}=k_{t} \nabla^{2} T$

$\rho=\rho_{0}\left[1-\alpha_{t}\left(T-T_{a}\right)\right]$.

Maxwell's equations in the magnetostatic limit:

$\nabla \cdot \vec{B}=0, \nabla \times \vec{H}=0, \vec{B}=\mu_{0}(\vec{M}+\vec{H})$

$\vec{M}=\left[M_{0}+\chi\left(H-H_{0}\right)-K\left(T-T_{0}\right)\right]\left(\frac{\vec{H}}{H}\right)$.

Here, $\vec{q}$ is the velocity, $p$ the pressure, $\rho$ the fluid density, $\vec{M}$ the magnetization, $\vec{H}$ the magnetic field intensity, $\vec{B}$ the magnetic flux density, $\mu_{0}$ the magnetic permeability of vacuum, $k_{t}$ the thermal conductivity, $C_{V}$, ${ }_{H}$ the specific heat at constant volume and magnetic field, $\rho_{0}$ the reference density, $\alpha_{t}$ the thermal expansion coefficient, $T_{a}=\left(T_{0}+T_{1}\right) / 2$ the average temperature, $M_{0}=M\left(H_{0}, T_{0}\right), \chi=(\partial M / \partial H)_{H_{0}, T_{0}}$ the magnetic susceptibility, $K=-(\partial M / \partial T)_{H_{0}, T_{0}}$ the pyromagnetic coefficient, $H=|\vec{H}|$ and $M=|\vec{M}|$.

The basic state is quiescent and given by

$\vec{q}=0, p=p_{b}(z), \eta=\eta_{b}(z),-\frac{d T_{b}}{d z}=f(z), \vec{H}_{b}=\left[H_{0}-\frac{K \beta z}{1+\chi}\right] \hat{k}$,

$\vec{M}_{b}=\left[M_{0}+\frac{K \beta z}{1+\chi}\right] \hat{k}$,

where, $\beta=\left(T_{l}-T_{u}\right) / d$ is the temperature gradient, $\hat{k}$ is the unit vector in the vertical direction and the subscript $b$ denotes the basic state. Following [14,19], the basic state cubic temperature profile is taken in the form

$T_{b}=T_{1}-a_{1}(z-d)-a_{2}(z-d)^{2}-a_{3}(z-d)^{3}$,

where, $a_{1}, a_{2}$ and $a_{3}$ are constants. The principle of exchange of stability is assumed to be valid as there are no physical mechanisms to set up oscillatory motions. Following the standard linear stability analysis procedure, the stability equations in dimensionless form can then be shown to be (for details see $[3,13,14])$ :

$$
\begin{aligned}
& (1+\Lambda)\left(D^{2}-a^{2}\right)^{2} W=\operatorname{Raa}^{2} \Theta-\operatorname{RaM}_{1} a^{2} f(z)(D \Theta-\Theta) \\
& \left(D^{2}-a^{2}\right) \Theta=-\left(1-M_{2}\right) f(z) W \\
& \left(D^{2}-a^{2} M_{3}\right) \Phi=D \Theta,
\end{aligned}
$$

where, $D=d / d z$ is the differential operator, $a=\sqrt{l^{2}+m^{2}}$ the overall horizontal wave number, $W$ the amplitude of vertical component of perturbed velocity, $\Theta$ the amplitude of perturbed temperature, $\Phi$ the amplitude of perturbed magnetic potential, $R a=\alpha_{t} g \beta d^{2} / \kappa v$ the thermal Rayleigh number, $M_{1}=\mu_{0} K^{2} \beta /(1+\chi) \alpha_{t} \rho_{0} g$ the magnetic number, $M_{2}=\mu_{0} T_{0} K^{2} / \rho_{0} C_{0}(1+\chi)$ the non-dimensional parameter $M_{3}=\left(1+M_{0} / H_{0}\right) /(1+\chi)$ the measure of nonlinearity of fluid magnetization parameter and $\Lambda=\delta \mu_{0}\left(M_{0}+H_{0}\right)$ the MFD viscosity parameter and the non-dimensional temperature gradient $f(z)$ is given by

$f(z)=a_{1}^{*}+2 a_{2}^{*}(z-1)+3 a_{3}^{*}(z-1)^{2}$.

The typical value of $M_{2}$ for magnetic fluids with different carrier liquids turns out to be of the order of $10^{-6}([3])$ and hence its effect is neglected as compared to unity. Three types of basic temperature gradients are considered for discussion as mentioned below ([14,19]):

\begin{tabular}{lllll}
\hline $\begin{array}{l}\text { Reference steady-state temperature } \\
\text { gradient }\end{array}$ & $f(z)$ & $a_{1}^{*}$ & $a_{2}^{*}$ & $a_{3}^{*}$ \\
Linear & 1 & 1 & 0 & 0 \\
Cubic 1 & $3(z-1)^{2}$ & 0 & 0 & 1 \\
Cubic 2 & $0.66+1.02(z-1)^{2}$ & 0.66 & 0 & 0.34 \\
\hline
\end{tabular}

The boundary conditions are:

$W=D W=\Theta=\Phi=0$ atz $=0$

$W=(1+\Lambda) D^{2} W+a^{2} M a \Theta=D \Theta+B i \Theta=\Phi=0$ atz $=1$,

where, $M a=\sigma_{T} \Delta T d / \mu \kappa$ is the Marangoni number $B i=h d / k_{t}$ is the Biot number. The case $B i=0$ and $B i \rightarrow \infty$ respectively correspond to constant heat flux and isothermal conditions at the upper boundary. 


\section{Method of solution}

Eqs. (9)-(11) together with the boundary conditions constitute an eigenvalue problem with $R_{t}$ or $\mathrm{Ma}$ as an eigenvalue. The eigenvalue problem is solved numerically using the Galerkin method with wave number as a perturbation parameter. Accordingly, the variables are written in a series of basis functions as

$W(z)=\sum_{i=1}^{n} A_{i} W_{i}(z), \Theta(z)=\sum_{i=1}^{n} B_{i} \Theta_{i}(z), \Phi(z)=\sum_{i=1}^{n} C_{i} \Phi_{i}(z)$

where, $A_{i}, B_{i}, C_{i}$ are unknown constants to be determined. The basis functions $W_{i}(z), \Theta_{i}(z), \Phi_{i}(z)$ are generally chosen such that they satisfy the corresponding boundary conditions but not the differential equations. For rigid ferromagnetic boundaries, they are chosen as

$W_{i}=\left(z^{4}-2 z^{3}+z^{2}\right) T_{i-1}^{*}, \Theta_{i}=\left(z-z^{2} / 2\right) T_{i-1}^{*}, \Phi_{i}=\left(z-z^{2}\right) T_{i-1}^{*}$,

where, $T_{i}^{*}$ ' $s$ are the modified Chebyshev polynomials. The above trial functions satisfy all the boundary conditions except, $(1+\Lambda)$ $D^{2} W+a^{2} M a \Theta=0=D \Theta+B i \Theta$ at $z=1$ but the residual from this is included as the residual from Eqs. (9)-(11). Multiplying momentum Eq. (9) by $W_{i}(z)$, energy Eq. (10) by $\Theta_{i}(z)$, magnetic potential Eq. (11) by $\Phi_{i}(z)$, performing the integration by parts with respect to $\mathrm{z}$ from $\mathrm{z}=0$ to $\mathrm{z}=1$ and using the boundary conditions, we obtain a system of linear homogeneous algebraic equations in $A_{i}, B_{i}, C_{i}$. A nontrivial solution exists if and only if the characteristic determinant is equal to zero. This leads to a relation

$f\left(R a, M a, \Lambda, B i, M_{1}, M_{3}, a_{1}^{*} a_{2}^{*}, a_{3}^{*}, a\right)=0$.

The critical values of $R a_{c}$ and $M a_{c}$ are determined numerically with respect to wave number $a_{c}$.

\section{Numerical results and discussion}

It may be noted that Eq. (16) leads to the characteristic equation giving the Marangoni number Ma or the thermal Rayleigh number $R a$ as a function of the wave number $a$, the parameters $R_{m}, B i, M_{1}, M_{3}, \Lambda$ and for different forms of basic temperature profiles (i.e., linear, cubic 1 and cubic 2 temperature profiles). Computations reveal that the convergence in finding the critical eigenvalue $\left(M a_{c}\right.$ or $\left.R a_{c}\right)$ crucially depends on the value of MFD viscosity parameter $\Lambda$ and various forms of basic temperature profiles. The Galerkin method is used to find the eigenvalues numerically as this technique is found to be more convenient to tackle different forms of basic temperature profiles. The results presented here are for $i=j=6$ the order at which the convergence is achieved, in general. To validate the numerical solution procedure used, a new magnetic parameter $S$, independent of the temperature gradient,

Table 1

Comparison of critical values of $R a_{c}$ and $R_{m c}$ for different values of $M a$ and $B i$ for $S=10^{-4}$ $M_{3}=1$ and $\Lambda=0$ with linear temperature profile $f(z)=1$.

\begin{tabular}{cccccc}
\hline \multirow{3}{*}{$B i$} & $M a$ & \multicolumn{2}{c}{ Qin and Kaloni [8] } \\
& \multicolumn{2}{c}{$a_{c} R_{m c}$} & \multicolumn{3}{c}{ Present analysis } \\
$R a_{c} R_{m c}$ & \\
\hline 0 & 0 & 652.87 & 42.624 & 649.84 & 42.229 \\
& 20 & 493.23 & 24.426 & 491.07 & 24.115 \\
& 40 & 335.98 & 11.255 & 335.38 & 11.248 \\
& 60 & 171.44 & 2.939 & 171.90 & 2.955 \\
& 70 & 85.67 & 0.734 & 85.74 & 0.735 \\
& 79.61 & 0.00 & 0.00 & 0.00 & 0.00 \\
& 0 & 892.06 & 79.577 & 903.55 & 81.640 \\
& 150 & 628.88 & 39.298 & 638.19 & 40.729 \\
& 250 & 418.23 & 17.492 & 427.29 & 18.258 \\
& 300 & 301.89 & 9.114 & 302.73 & 9.405 \\
& 350 & 176.10 & 3.101 & 177.74 & 3.158 \\
& 413.4 & 0.00 & 0.00 & 0.00 & 0.00 \\
\hline
\end{tabular}

was introduced in the form $R_{m}=R a^{2} S$, where $S=\mu_{0} K^{2} \kappa \nu$ / $(1+\chi) \rho_{0} g^{2} \alpha^{2} d^{4}$. The critical thermal Rayleigh number $\left(R a_{c}\right)$ and the critical magnetic Rayleigh number $\left(R_{m c}\right)$ obtained for different values of Marangoni number $M a$ and for linear temperature profile (i.e., $a_{1}^{*}=1, a_{2}^{*}=0$ and $a_{3}^{*}=0$ ) are exhibited in Table 1 when $S=10^{-4}$ and $\Lambda=0$ along with the results of Qin and Kaloni [8]. We note that there is a close agreement between the results of present analysis and those obtained by Qin and Kaloni [8] using different numerical approach (see Table 1). The values of magnetic parameters chosen are based on the physical parameters for a commercially available magnetic fluid EMG 905 produced by Ferrofluidics [20]; density $\rho\left[\mathrm{kg} / \mathrm{m}^{3}\right]=1.24 \times 10^{3}$, kinematic viscosity $\left(27{ }^{\circ} \mathrm{C}\right)$ $\nu\left[\mathrm{m}^{2} / \mathrm{s}\right]=12 \times 10^{-6}$, thermal diffusivity $\kappa\left[\mathrm{m}^{2} / \mathrm{s}\right]=8 \times 10^{-8}$, heat capacity $c_{p}[\mathrm{~J} / \mathrm{kgK}]=1.47 \times 10^{3}$, coefficient of thermal expansion $\alpha_{t}[1 / \mathrm{K}]=8.6 \times 10^{-4}$, susceptibility at low field $\chi=1.9$, pyromagnetic coefficient at $H=50 \mathrm{kA} / \mathrm{m}[\mathrm{A} / \mathrm{km}]=110$ and mean particle diameter $[\mathrm{nm}]=10.2$. For such fluids the magnetic parameters have the following order of magnitude $M_{1} \sim 10^{-4}-10$ and $M_{3} \geq 1$.
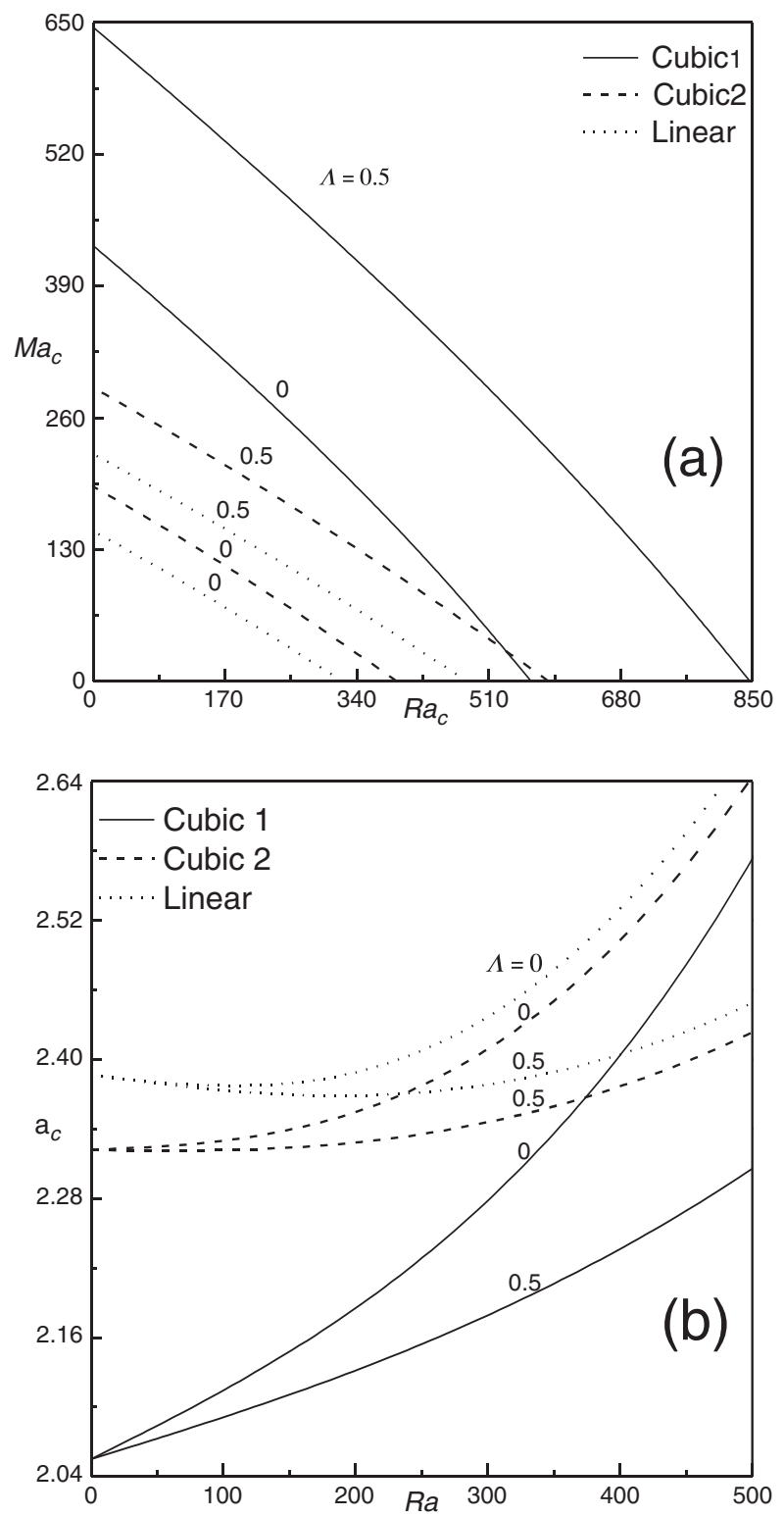

Fig. 1. (a). Locus of $M a_{c}$ and $R a_{c}$ for different values of $\Lambda$ for $B i=2, M_{1}=2$ and $M_{3}=1$.(b). Variation of $a_{c}$ verses Ra for different values of $\Lambda$ for $B i=2, M_{1}=2$ and $M_{3}=1$. 

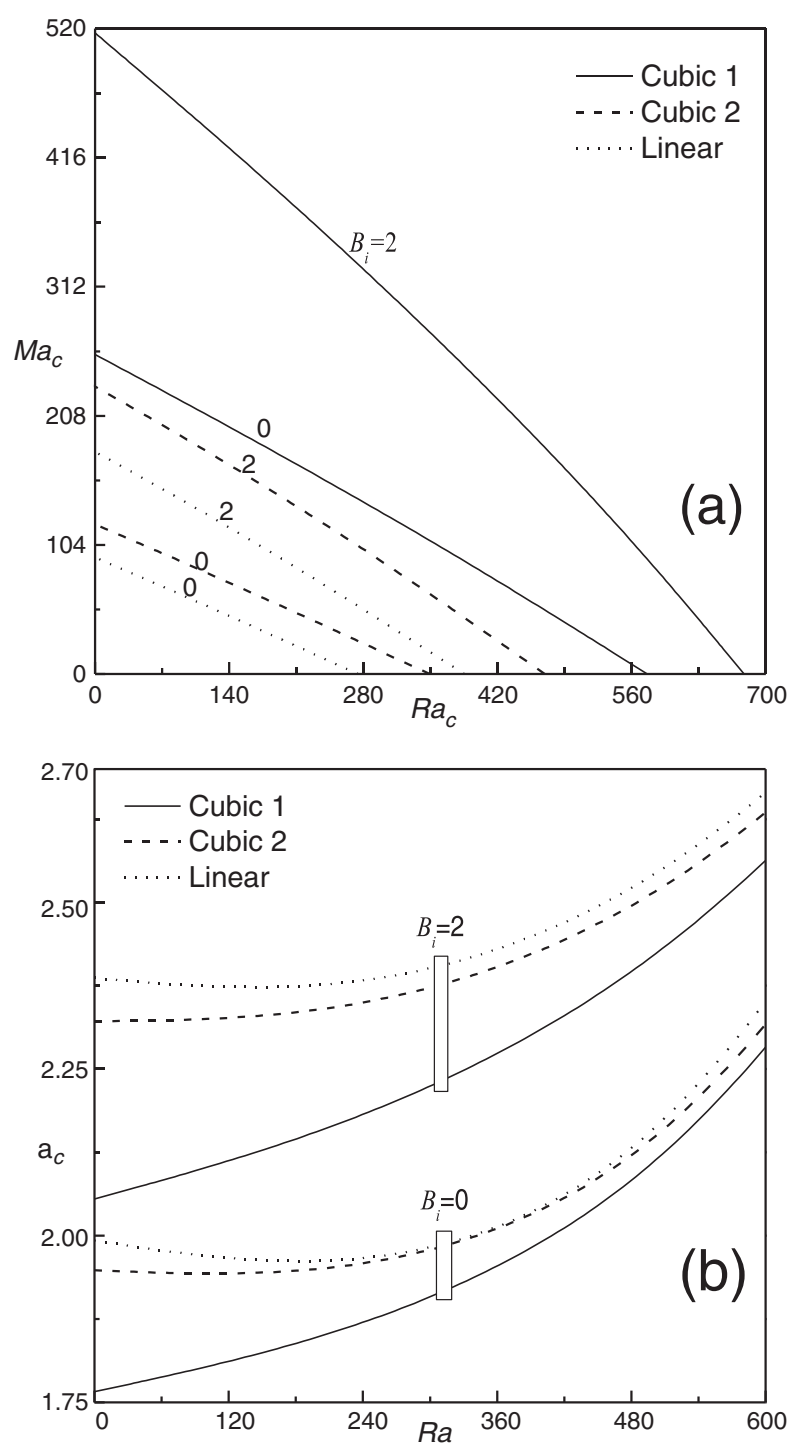

Fig. 2. (a). Locus of $M a_{c}$ and $R a_{c}$ for different values of $B i$ for $\Lambda=0.2, M_{1}=2$ and $M_{3}=1$.(b). Variation of $a_{c}$ verses $R a$ for different values of $B i$ for $\Lambda=0.2, M_{1}=2$ and $M_{3}=1$.

We now look into the solution of the complete problem, which involves the effect of all the parameters $R a, M a, R_{m}, B i, \Lambda, M_{1}$ and $M_{3}$ on the criterion for the onset of ferroconvection. The salient characteristics of these parameters are exhibited graphically in Figs. 1-7. These figures exhibit a tight coupling between the buoyancy, magnetic and surface tension forces. Fig. 1(a) shows the locus of the critical Marangoni number $M a_{c}$ and the Rayleigh number $R a_{c}$ for different values of MFD viscosity parameter $\Lambda$ for $B i=2, M_{1}=2$ and $M_{3}=1$ for different forms of basic temperature profiles. It is seen that the curves are slightly convex and an increase in the Rayleigh number has a destabilizing effect on the system. That is, buoyancy and surface tension forces complement with each other. Also, an increase in the MFD viscosity parameter has a stabilizing effect on the system. Moreover, $\left(M a_{c} \text { or } R a_{c}\right)_{\text {linear }}<\left(M a_{c}\right.$ or $\left.R a_{c}\right)_{\text {cubic } 2}<\left(M a_{c} \text { or } R a_{c}\right)_{\text {cubic } 1}$ suggesting that the cubic 1 temperature profile is more stabilizing than cubic 2 and the linear temperature profile is the least stable. Thus, it is possible to control Bénard-Marangoni ferroconvection effectively by the choice of different forms of basic temperature profiles. The variation in $a_{c}$ as a function of $R a$ is elucidated in Fig. 1(b) for different forms of basic temperature profile with different values of $\Lambda$ It may be noted that the critical wave number $a_{c}$ increases monotonically with increasing $R a$. Moreover, an increase in the value of
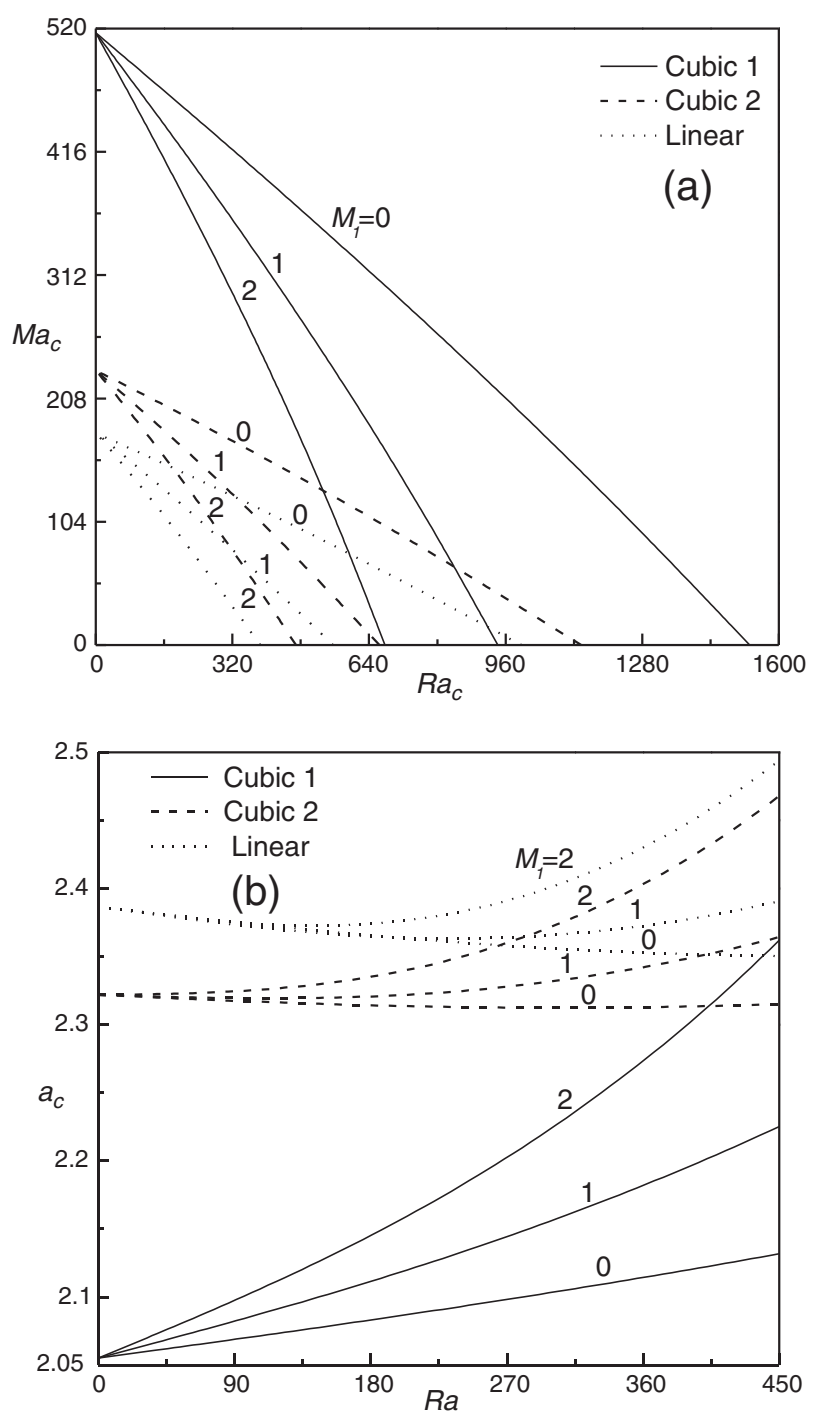

Fig. 3. (a). Locus of $M a_{c}$ and $R a_{c}$ for different values of $M_{1}$ for $\Lambda=0.2, B i=2$ and $M_{3}=1$.(b). Variation of $a_{c}$ verses $R a$ for different values of $M_{1}$ for $\Lambda=0.2, B i=2$ and $M_{3}=1$.

$\Lambda$ is to decrease $a_{c}$ and thus its effect is to reduce the size of convection cells and also $\left(a_{c}\right)_{\text {cubic } 1}<\left(a_{c}\right)_{\text {cubic } 2}<\left(a_{c}\right)_{\text {linear }}$.

The plots in Fig. 2(a) represent the locus of $M a_{c}$ and $R a_{c}$ for different values of heat transfer coefficient $B i$ (i.e., Biot number) when $\Lambda=0.2$, $M_{3}=1$ and $M_{1}=2$ for three different forms of basic temperature profiles. From the figure it is evident that an increase in the value of $B i$ is to increase $R a_{c}$ as well as $M a_{c}$ and thus its effect is to delay the onset of Bénard-Marangoni ferroconvection. This may be attributed to the fact that with increasing $B i$, the thermal disturbances can easily dissipate into the ambient surrounding due to a better convective heat transfer coefficient at the top surface and hence higher heating is required to make the system unstable. Fig. 2(b) represents the corresponding critical wave number $a_{c}$ and it indicates that increase in the value of $B i$ and $R a$ is to increase $a_{c}$ and thus their effect is to reduce the size of convection cells.

Fig. 3(a) presents the locus of $R a_{c}$ and $M a_{c}$ for different forms of basic temperature profiles and for various values of magnetic number $M_{1}$ when $\Lambda=0.2, M_{3}=1$ and $B i=2$. The results for $M_{1}=0$ correspond to ordinary viscous fluid and it is observed that higher heating is required to have instability in this case. Besides, the curves of different $M_{1}$ become closer as the value of $M_{1}$ increases. Thus the combined effect of surface tension, magnetic and buoyancy forces is to reinforce together 

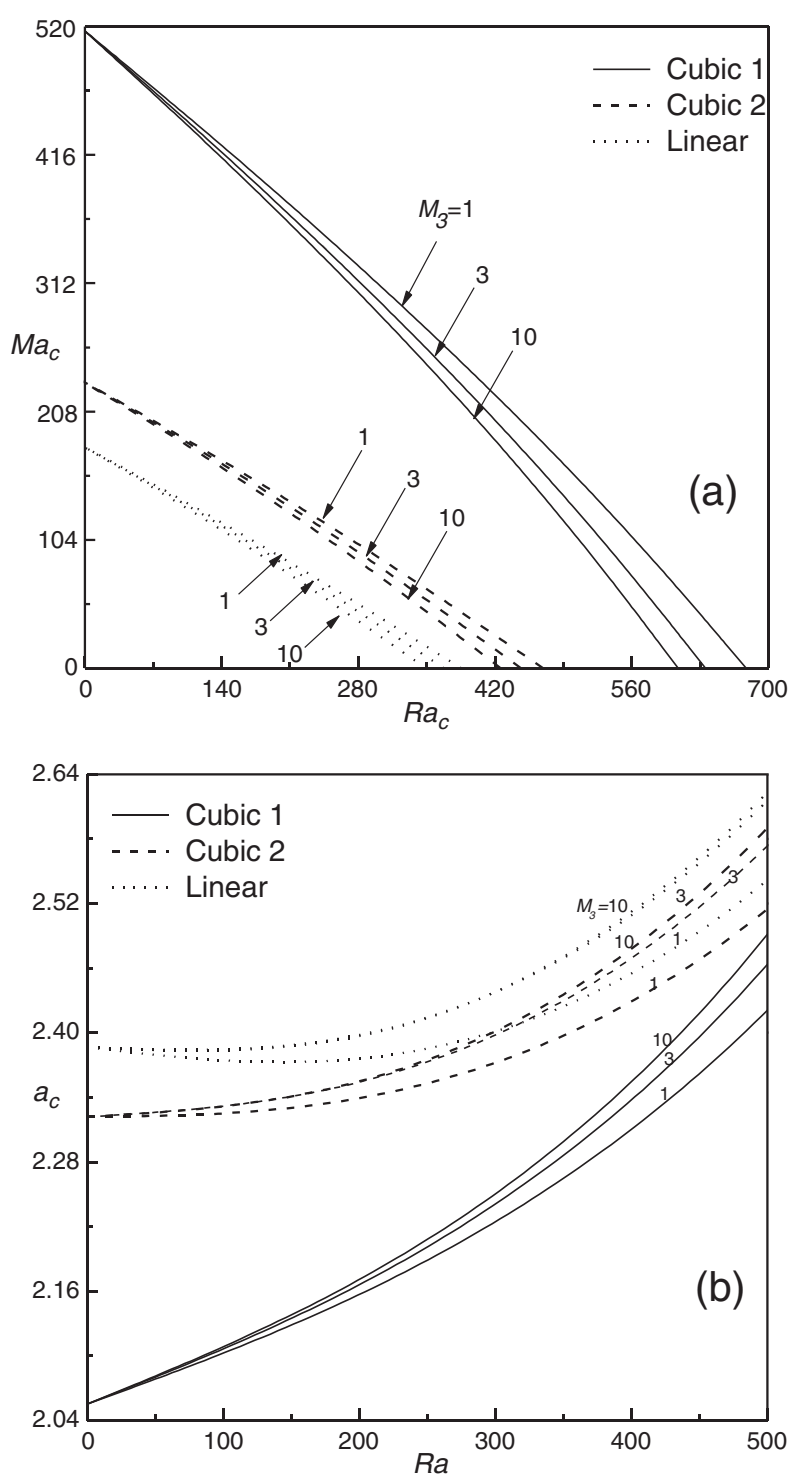

Fig. 4. (a). Locus of $M a_{c}$ and $R a_{c}$ for different values of $M_{3}$ for $\Lambda=0.2, B i=2$ and $M_{1}=2$.(b). Variation of $a_{c}$ verses $R a$ for different values of $M_{3}$ for $\Lambda=0.2, B i=2$ and $M_{1}=2$.

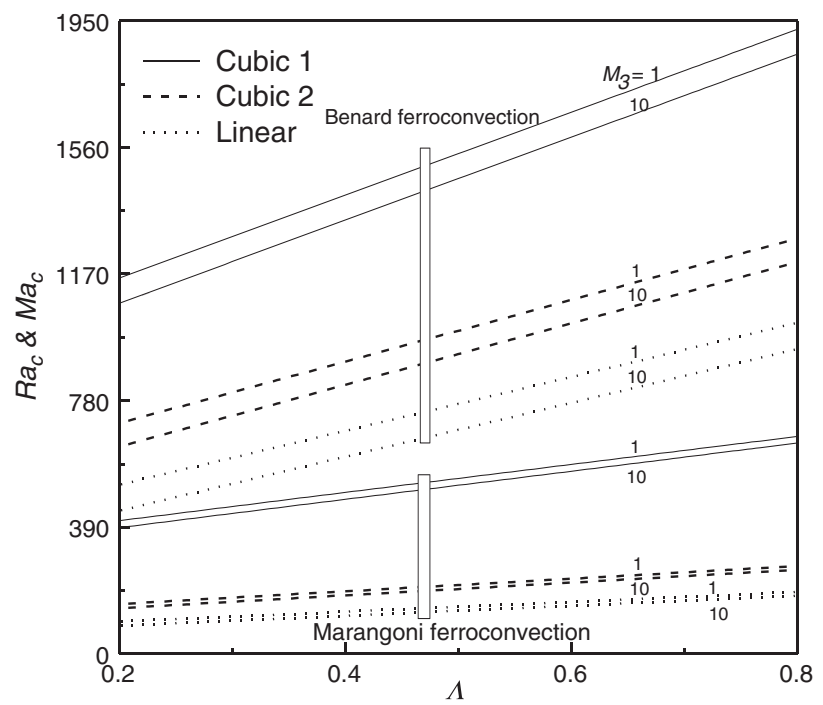

Fig. 5. Variation of $M a_{c}$ and $R a_{c}$ verses $\Lambda$ with two values of $M_{3}$ for $R_{m}=100$ and $B i=2$.

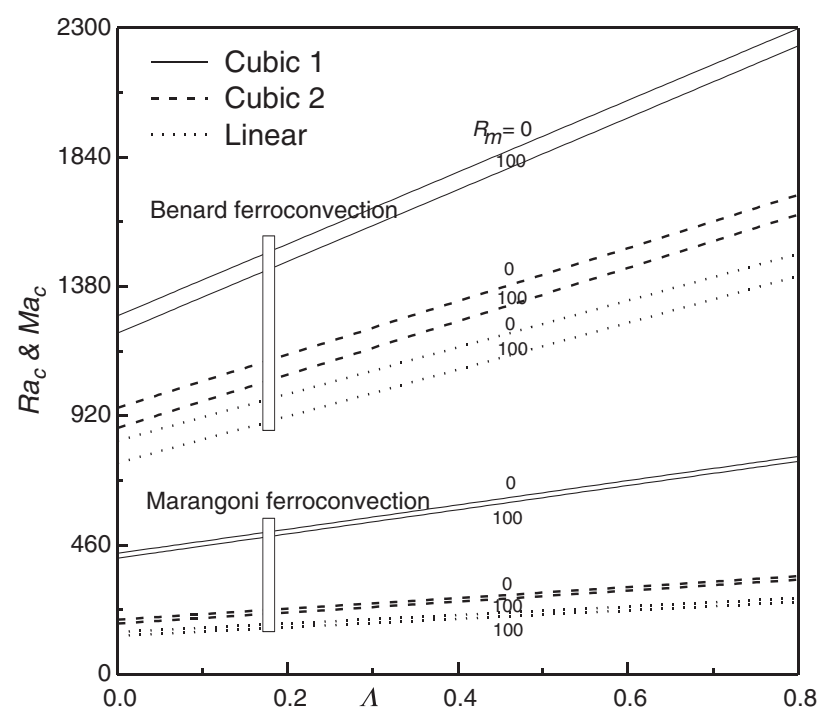

Fig. 6. Variation of $M a_{c}$ and $R a_{c}$ verses $\Lambda$ with two values of $R_{m}$ for $B i=2$ and $M_{3}=1$.

and to hasten the onset of Bénard-Marangoni ferroconvection compared to their effect in isolation. Although the critical wave number $a_{c}$ remains invariant for different values of $M_{1}$ at lower values of $R a$, it increases with further increase in the value of $R a$ (see Fig. 3(b)). Further inspection of Fig. 3(b) shows that the cubic 1 basic temperature profile is more stabilizing when compared to the other two profiles. The deviation in the critical wave number amongst different values of $M_{1}$, with increasing $M_{1}$ as well as $R a$, is to increase the critical wave number $a_{c}$ and hence to reduce the size of the convection cells.

The measure of non-linearity of fluid magnetization, denoted through the parameter $M_{3}$, on the onset of ferroconvection in a ferrofluid layer is depicted in Fig. 4(a). The curves of $M a_{c}$ as a function of $R a_{c}$ are shown in Fig. 4(a) for different values of $M_{3}$ are for $\Lambda=0.2$, $B i=2$ and $M_{1}=2$. It can be seen that an increase in $M_{3}$ is to decrease $R a_{c}$ and $M a_{c}$ but only marginally and thus it has a destabilizing effect on the system. This may be due to the fact that the application of magnetic field makes the ferrofluid to acquire larger magnetization which in turn interacts with the imposed magnetic field and releases more energy to drive the flow faster. Hence, the system becomes unstable with a smaller temperature gradient as the value of $M_{3}$ increases. Alternatively, a

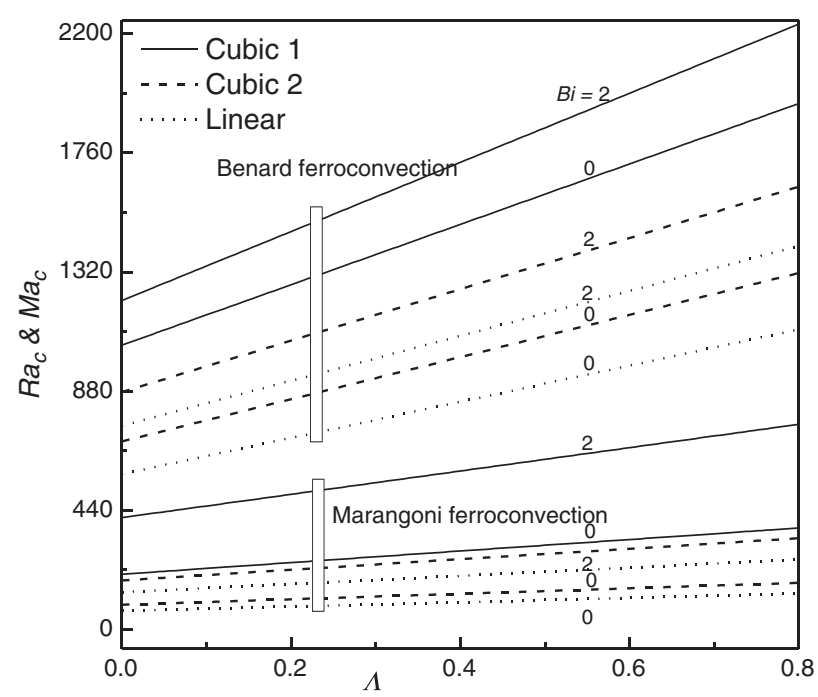

Fig. 7. Variation of $M a_{c}$ and $R a_{c}$ verses $\Lambda$ with two values of $B i$ for $M_{3}=1$ and $R_{m}=100$. 
higher value of $M_{3}$ would arise either due to a larger pyromagnetic coefficient or larger temperature gradient. Both these factors are conducive for generating a larger gradient in the Kelvin body force field, possibly promoting the instability. The variation of $a_{c}$ as a function of $R a$ is shown in Fig. 4(b) for different values of $M_{3}$. From the figure, we note that increasing $M_{3}$ and $R a$ is to increase $a_{c}$ and hence to decrease the dimension of convection cells.

Figs. 5-7 show the isolation presence of surface tension and buoyancy forces on the onset of ferroconvection. These figures illustrate the variation of $M a_{c}$ (pure Marangoni ferroconvection, $R a=0$ ) and $R a_{c}$ (pure Bénard ferroconvection, $M a=0$ ) as a function of $\Lambda$ for different values of physical parameters $M_{3}, R_{m}$ and $B i$ for three types of basic temperature profiles. From the figures, it is seen that the effect of increasing $\Lambda$ is the delay of the onset of Bénard/Marangoni ferroconvection and also an increase in $M_{3}$ (Fig. 5), $R_{m}$ (Fig. 6) and a decrease in Bi (Fig. 7) which decreases the critical Rayleigh/Marangoni number and hastens the onset of ferroconvection. Further, $\left(M a_{c}\right)_{\text {linear }<\text { cubic } 2<\text { cubic } 1}<\left(R a_{c}\right)_{\text {linear }<\text { cubic } 2<\text { cubic } 1 \text { as observed }}$ in ordinary viscous fluids.

\section{Conclusions}

The buoyancy, surface tension and magnetic forces reinforce each other to hasten the onset of ferroconvection. It is demonstrated that the onset of Bénard-Marangoni ferroconvection can be controlled effectively by the proper choice of basic temperature profiles. The cubic 1 basic temperature profile delays, while linear profile hastens the onset of Bénard-Marangoni ferroconvection. Besides, an increase in the value of $\Lambda, B i$ and a decrease in $R_{m}$ and $M_{3}$ delays the onset of Bénard-Marangoni ferroconvection. The dimension of convection cells decreases with increasing $B i$, and $\Lambda$ as well as decrease in $M_{1}$ and $M_{3}$. The critical wave numbers for the cubic 1 basic temperature profile are lower than those of the cubic 2 and linear temperature profiles. The critical Marangoni numbers are lower than the critical Rayleigh numbers for all temperature profiles considered.

\section{Acknowledgments}

The authors gratefully acknowledge the financial assistance under the VTU research grant scheme, received from the Visvesvaraya Techno- logical University (Letter No. VTU./Aca./2011-12/A-9/766), Belgaum, Karnataka State.

\section{References}

[1] R.E. Rosensweig, Ferrohydrodynamics, Cambridge University Press, Cambridge, 1985.

[2] S. Odenbach, Ferrofluids: Magnetically Controllable Fluids and Their Applications, Springer, Berlin, 2003.

[3] B.A. Finlayson, Convective instability of ferromagnetic fluids, J. Fluid Mech. 40 (1970) 753-767.

[4] P.J. Stiles, M.J. Kagan, Thermoconvective instability of a ferrofluid in a strong magnetic field, J. Colloid Interface Sci. 134 (1990) 435-449.

[5] R. Ganguly, S. Sen, I.K. Puri, Heat transfer augmentation using a magnetic fluid under the influence of a line dipole, J. Magn. Magn. Mater. 271 (2004) 63-73.

[6] C.E. Nanjundappa, I.S. Shivakumara, Effect of velocity and temperature boundary conditions on convective instability in a ferrofluid layer, ASME J. Heat Transf. 130 (2008) 1045021-1045025.

[7] I.S. Shivakumara, Jino Lee, C.E. Nanjundappa, Onset of thermogravitational convection in a ferrofluid layer with temperature dependent viscosity, ASME J. Heat Transf. 134 (2012) 0125011-0125017.

[8] Y. Qin, P.N. Kaloni, Nonlinear stability problem of a ferromagnetic fluid with surface tension effect, Eur. J. Mech. B Fluids 13 (1994) 305-321.

[9] J. Weilepp, H.R. Brand, Competition between the Bénard-Marangoni and the Rosensweig instability in magnetic fluids, J. Phys. II France 6 (1996) 419-441.

[10] S. Odenbach, On the stability of a free surface of a magnetic fluid under microgravity, Adv. Space Res. 22 (1998) 1169-1173.

[11] I.S. Shivakumara, N. Rudraiah, C.E. Nanjundappa, Effect of non-uniform basic temperature gradient on Rayleigh-Bénard-Marangoni convection in ferrofluids, J. Magn. Magn. Mater. 248 (2002) 379-395.

[12] I.S. Shivakumara, C.E. Nanjundappa, Marangoni ferroconvection with different initial temperature gradients, J. Energy Heat Mass Transfer 28 (2006) 45-61.

[13] C.E. Nanjundappa, I.S. Shivakumara, R. Arunkumar, Bénard-Marangoni ferroconvection with magnetic field dependent viscosity, J. Magn. Magn. Mater. 322 (2010) 2256-2263.

[14] R. Idris, I. Hashim, Effects of controller and cubic temperature profile on onset of Bénard-Marangoni convection in ferrofluid, Int. Commun. Heat Mass Transf. 37 (2010) 624-628

[15] C.E. Nanjundappa, I.S. Shivakumara, R. Arunkumar, Onset of Bénard-Marangon ferroconvection with internal heat generation, Microgravity Sci. Technol. 23 (2011) 29-39.

[16] C.E. Nanjundappa, I.S. Shivakumara, R. Arunkumar, Onset of Marangoni-Bénard ferroconvection with temperature dependent viscosity, Microgravity Sci. Technol. 25 (2013) 103-112.

[17] R.E. Rosenwieg, R. Kaiser, G. Miskolczy, Viscosity of magnetic fluid in a magnetic field, J. Colloid Interface Sci. 29 (4) (1969) 680-686.

[18] G. Vaidyanathan, R. Sekar, A. Ramanathan, Effect of magnetic field dependent viscosity on ferroconvection in rotating medium, Indian J. Pure Appl. Phys. 40 (2002) 159-165.

[19] K.T. Chiang, Effect of a non-uniform basic temperature gradient on the onset of Bénard-Marangoni convection: stationary and oscillatory analyses, Int. Commun. Heat Mass Transf. 32 (2005) 192-203.

[20] G.K. Auernhammer, H.R. Brand, Thermal convection in a rotating layer of a magnetic fluid, Eur. Phys. J. B. 16 (2000) 157-168. 